\title{
A Counterexample in Hardy Spaces with an Application to Systems Theory
}

\author{
V. Katsnelson and G. Weiss
}

\begin{abstract}
We know from elementary calculus that if $f$ is a differentiable function on $(0, \infty)$ with $f(\infty)=0$, then $\lim _{x \rightarrow+\infty} x f(x)=-\lim _{x \rightarrow+\infty} x^{2} f^{\prime}(x)$ in the following sense: if the second limit exists, then so does the first and they are equal. The existence of the first limit does not imply the existence of the second, in general. It was hoped that the existence of the first limit, together with the assumption that $f$ belongs to the Hardy class $H^{2}$, does imply the existence of the second limit. This would have simplified certain results in systems theory. We show by counterexample that this is not the case. More precisely, for each $q>1$ (including $q=\infty$ ) we construct a function $f$ in the Hardy space $H^{q}$ on the right half-plane such that $\lim _{x \rightarrow+\infty} x f(x)=0$ and $\lim \sup _{x \rightarrow+\infty} x^{2}\left|f^{\prime}(x)\right|=\infty$. A special Blaschke product plays a crucial role in this construction.

Keywords: Hardy classes, Blaschke products, Tauberian theorems, infinite dimensional systems, observation operators
\end{abstract}

AMS subject classification: $30 \mathrm{D} 55,30 \mathrm{D} 50,93 \mathrm{C} 25$

\section{Background and formulation of the problem}

Let $f$ be a differentiable function on $(0, \infty)$ with $\lim _{x \rightarrow+\infty} f(x)=0$. Then by L'Hopital's rule

$$
\lim _{x \rightarrow+\infty} x f(x)=-\lim _{x \rightarrow+\infty} x^{2} f^{\prime}(x)
$$

holds, in the following sense: if the second limit exists (as a finite number), then also the first limit exists and they are equal. The existence of the first limit does not imply the existence of the second, as the simple example $f(x)=\frac{\sin x^{2}}{x^{2}}$ shows.

The following question arose in systems theory: Suppose $f$ belongs to the Hardy space $H^{2}$ on the right half-plane. Then does the existence of the first limit in (1.1) guarantee the existence of the second limit (and hence the equality (1.1))? More details about the connection with systems theory will be given in Section 2 .

The aim of this paper is to give a negative answer to the above question, which seems to necessitate rather subtle techniques. In fact, we prove a slightly more general result, by considering instead of $H^{2}$ the Hardy spaces $H^{p}$, with $1 \leq p \leq \infty$. It turns

V. Katsnelson: The Weizmann Inst. of Science, Dep. Theor. Math., Rehovot 76100, Israel G. Weiss: Ben-Gurion Univ. of the Negev, Dep. Electr. Engin., Beer Sheva 84105, Israel 
out that the answer is negative for all $p>1$ and positive for $p=1$ (for $p=1$ both sides of (1.1) are zero).

We denote by $\mathbb{C}_{+}$the open right half-plane in $\mathbb{C}$. Let us recall the basic facts about the Hardy classes $H^{p}$ on $\mathbb{C}_{+}$, where $1 \leq p \leq \infty$. For more details the reader may consult, for example, Duren [4]. First suppose $p<\infty$. By definition, $f \in H^{p}$ if $f$ is analytic on $\mathbb{C}_{+}$and

$$
\sup _{0<x<\infty} \int_{-\infty}^{+\infty}|f(x+i y)|^{p} d y<\infty
$$

If we define $\|f\|^{p}$ as the expression in (1.2), then $H^{p}$ becomes a Banach space. If $f \in H^{p}$, then for almost every real $y$, the limit $f(i y):=\lim _{\varepsilon \downarrow 0} f(\varepsilon+i y)$ exists and

$$
\|f\|^{p}=\int_{-\infty}^{+\infty}|f(i y)|^{p} d y .
$$

Moreover, $f$ is representable by means of the Cauchy integral

$$
f(s)=\frac{-1}{2 \pi} \int_{-\infty}^{+\infty} f(i \omega) \frac{1}{i \omega-s} d \omega .
$$

Formula (1.3) admits differentiation with respect to $s$ :

$$
f^{\prime}(s)=\frac{-1}{2 \pi} \int_{-\infty}^{+\infty} f(i \omega) \frac{1}{(i \omega-s)^{2}} d \omega
$$

Now we turn to the case $p=\infty$. The space $H^{\infty}$ consists of all bounded analytic functions on $\mathbb{C}_{+}$and it is a Banach space with the sup norm. The boundary function $y \rightarrow f(i y)$ can be defined almost everywhere, as in the case $p<\infty$, and its essential supremum equals $\|f\|$. Formula (1.3) does not make sense in general.

It is casy to derive the affirmative answer to the problem mentioned earlier, for the case $p=1$. Indeed, if $f \in H^{1}$, then from the integral representation (1.3) and from the Lebesgue dominated convergence theorem (take into account that $\int_{-\infty}^{+\infty}|f(i y)| d y<\infty$ ) it follows that

$$
\lim _{x \rightarrow+\infty} x f(x)=\int_{-\infty}^{+\infty} f(i y) d y .
$$

Analogously, from the integral representation (1.4) it follows that

$$
\lim _{x \rightarrow+\infty} x^{2} f^{\prime}(x)=-\int_{-\infty}^{+\infty} f(i y) d y .
$$


Thus for a function $f \in H^{1}$ the limits in (1.5) and (1.6) exist and moreover they are both zero, because

$$
\int_{-\infty}^{+\infty} f(i y) d y=0
$$

Therefore, (1.1) is true for all $f \in H^{1}$.

If $f \in H^{p}$ with $p>1$, then the limit in (1.5) need not exist. If it exists, then it may be non-zero, as the simple example $f(s)=\frac{1}{s+1}$ shows. Now let us state our negative result for $p>1$ in precise terms. Our main result is the following

Theorem 1.1. For every $p \in(1, \infty)$ there exists an analytic function $f_{p}$ defined on $\mathbb{C}_{+}$such that

(i) $f_{p} \in H^{q}$ for all $q \geq p$ (including $q=\infty$ )

(ii) $\lim _{x \rightarrow+\infty} x f_{p}(x)=0$

(iii) $f_{p}(x)$ is real for real $x$

(iv) $\lim \sup _{x \rightarrow+\infty} x^{2} f_{p}^{\prime}(x)=\infty$ and $\liminf \sin _{x \rightarrow+\infty} x^{2} f_{p}^{\prime}(x)=-\infty$.

We give a constructive proof of this theorem in Sections $3-6$.

A result stating that the existence of the first limit in (1.1) together with some mild condition on $f$ implies the existence of the second limit in (1.1) is Tauberian by its nature. The mild assumption on $f$ is called a Tauberian condition. Our counterexample shows that the condition $f \in H^{p}$, with $p>1$, is not a Tauberian condition. We have already seen that $f \in H^{1}$ is a valid Tauberian condition. The following proposition gives another example of a Tauberian condition: $f$ should have an analytic extension to an angular domain around the positive real axis and the first limit in (1.1) should be valid in this angular domain. This is satisfied, for example, by any rational function for which the first limit in (1.1) exists. We need a notation for angular domains in $\mathbb{C}_{+}$: for any $\psi \in\left(0, \frac{\pi}{2}\right)$,

$$
\mathcal{W}(\psi)=\left\{r e^{i \phi} \mid r \in(0, \infty), \phi \in(-\psi,+\psi)\right\} .
$$
If

Proposition 1.2. Suppose $f$ is an analytic function on an angular domain $\mathcal{W}(\psi)$.

$$
\lim _{|s| \rightarrow \infty, s \in \mathcal{W}(\psi)} s f(s)=L
$$

with $L$ finite, then for any $\phi<\psi$

$$
\lim _{|s| \rightarrow \infty, s \in \mathcal{W}(\phi)} s^{2} f^{\prime}(s)=-L .
$$

Proof. For any $\varepsilon>0$ and any $s \in C$ denote $C_{\varepsilon}(s)=\{\zeta \in C:|\zeta-s|=\varepsilon|s|\}$ (this is a circle around $s$ ). We can find an $\varepsilon>0$ sufficiently small such that $C_{\varepsilon}(s) \subset \mathcal{W}(\psi)$ for all $s \in \mathcal{W}(\phi)$. Then by the Cauchy formula

$$
f^{\prime}(s)=\frac{1}{2 \pi i} \int_{C_{e}(s)} \frac{f(\zeta)}{(\zeta-s)^{2}} d \zeta .
$$

Since $f(\zeta)=\frac{L}{\zeta}(1+\rho(\zeta))$ where $\lim _{|\zeta| \rightarrow \infty} \rho(\zeta)=0$, substituting into (1.9) we can obtain $(1.8)$ 
The following proposition gives yet another valid Tauberian condition: $f$ should have an analytic extension to $\mathbb{C}_{+}$and its real part should be positive (such functions occur often in electrical circuit theory).

Proposition 1.3. Suppose $f$ is an analytic function on $\mathbb{C}_{+}$such that $\operatorname{Re} f(s) \geq 0$ and $f$ is not identically zero. If for $x>0$

$$
\liminf _{x \rightarrow+\infty}|x f(x)|<\infty
$$

then, for any $0<\psi, \phi<\frac{\pi}{2},(1.7)$ and (1.8) hold, with $0<L<\infty$.

Proof. If $g$ is an analytic function from the open unit disk $D$ to $\mathbb{C}_{+}$, then there is a unique finite positive Borel measure $\mu$ on $[0,2 \pi)$ such that

$$
g(z)=\int_{0}^{2 \pi} \frac{e^{i \alpha}+z}{e^{i \alpha}-z} d \mu(\alpha)+i \operatorname{Im} g(0) .
$$

Indeed, this follows from the Herglotz representation of positive harmonic functions on the disk, see Duren [4: p. 3] or Rudin [5: p. 262]. If $f$ is analytic from $\mathbb{C}_{+}$to itself, then $f(s)=g\left(\frac{s-1}{s+1}\right)$, where $g$ is as above. Doing a little computation, we get the integral representation (valid for all $s \in C_{+}$)

$$
f(s)=c s+\int_{-\infty}^{+\infty}\left(\frac{1}{s-i \omega}-\frac{i \omega}{1+\omega^{2}}\right)\left(1+\omega^{2}\right) d \sigma(\omega)+i d
$$

where $c \geq 0, d \in \mathbb{R}$ (in fact $c=\mu(\{0\})$ and $d=\operatorname{Im} g(0)$ ) and $\sigma$ is a finite positive Borel measure on $\mathbb{R}$ (see also Achieser and Glasmann [1: Section 69]

Until here we did not use (1.10). From (1.11) we see that $\operatorname{Re}(f(s)-c s)$ is the Poisson integral of $\left(1+\omega^{2}\right) d \sigma(\omega)$. Now (1.10) implies that $c=0$ and

$$
\int_{-\infty}^{+\infty}\left(1+\omega^{2}\right) d \sigma(\omega)=L<\infty
$$

From here we see that $\lim _{x \rightarrow+\infty} f(x)$ exists and again by (1.10) this limit must be zero. Thus we obtain the simple representation (valid for all $s \in C_{+}$)

$$
f(s)=\int_{-\infty}^{+\infty} \frac{1+\omega^{2}}{s-i \omega} d \sigma(\omega)
$$

Now (1.7) and (1.8) follow from this representation, together with (1.12)

Here is an alternative short proof for Proposition 1.3: By the theorem of Caratheodory, Julia and Wolff (see Burckel [2: pp. $203-206]$ ), $f$ satisfies $(1.7)$ for any $\psi \in\left(0, \frac{\pi}{2}\right)$. Now we can apply Proposition 1.2.

Our results are related to the following Tauberian theorem proved in Weiss [9]. 
Theorem 1.4. Let $W$ be a Banach space and $1<p<\infty$. Suppose that $v \in$ $L^{p}([0, \infty), W)$ is such that

$$
\sup _{t>0} \frac{1}{t} \int_{0}^{t}\|v(\sigma)\|^{p} d \sigma<\infty
$$

Let $\hat{v}$ denote the Laplace transform of $v$. Then, with $\lambda$ positive,

$$
\lim _{t \rightarrow 0} \frac{1}{t} \int_{0}^{t} v(\sigma) d \sigma=\lim _{\lambda \rightarrow \infty} \lambda \hat{v}(\lambda)
$$

i.e., if one of the limits exists, so does the other and they are equal.

We mention that if the limit on the left is known to exist, then, by a well known Abelian theorem, (1.14) is true regardless if (1.13) holds. The interesting case is when only the limit on the right is known to exist. In [9] the following question was raised: Is it possible to omit the condition (1.13) from Theorem 1.4? For $p \in(1,2)$ this question was answered in the negative in the same paper, via a family (indexed by $p$ ) of scalar counterexamples. The most important case, $p=2$, as well as $p \in(2, \infty)$, was left open in [9]. These cases can now be settled, also in the negative, using the main result of this paper. For $p=1$, Theorem 1.4 is not true, see Remark 5.9 in [9]. For $p=\infty$ the condition (1.13) becomes meaningless but (1.14) holds anyway, see again Remark 5.9 in [9]. For other Tauberian theorems about the Laplace transformation we refer to Doetsch [3].

Proposition 1.5. For any $p \in[2, \infty)$ there exists a function $v_{p} \in L^{p}[0, \infty)$ such that the limit on the right-hand side of (1.14) exists, but the limit on the left-hand side does not exist.

Proof. Let $q \in(1,2)$ be such that $\frac{1}{p}+\frac{1}{q}=1$. Let $v_{p}$ be the inverse Laplace transform of $f_{q}$ from Theorem 1.1. We have $v_{p} \in L^{p}[0, \infty)$ by the Hausdorff-Young theorem (see, e.g., [5: p. 277]). It is clear (by the definition of $v_{p}$ ) that the limit on the right of (1.14) is zero. If the limit on the left would exist, then by a simple Abelian theorem (see, for example, Proposition 5.4 in [9]) $s f_{q}(s)$ would have an angular limit as in (1.7), for any $\psi \in\left(0, \frac{\pi}{2}\right)$. By Proposition 1.2 it would follow that $x^{2} f_{q}^{\prime}(x)$ has a limit for $x \rightarrow+\infty$, which is a contradiction (according to Theorem 1.1)

\section{The connection with systems theory}

We reproduce three definitions from Weiss [8] and [9]. Later in this section we shall explain the role of these concepts in infinite dimensional systems theory.

Let $\mathrm{T}$ be a strongly continuous semigroup of operators on the Hilbert space $X$ and let $A$ (with domain $D(A)$ ) denote its generator. We define the Hilbert space $X_{1}$ as $D(A)$ with the norm $\|\cdot\|_{1}=\|(\beta I-A) \cdot\|$, where $\beta \in \rho(A)$ is fixed. This norm is equivalent to the graph norm. 
Definition 2.1. Suppose $Y$ is a Hilbert space. Then $C \in \mathcal{L}\left(X_{1}, Y\right)$ is called an admissible observation operator for $\mathbb{T}$ if for some (hence for any) $\tau \geq 0$ the estimate

$$
\int_{0}^{r}\left\|C \mathbb{T}_{\imath} x_{0}\right\|^{2} d t \leq M_{r}\left\|x_{0}\right\|^{2}
$$

holds, with $M_{\tau} \geq 0$ independent of $x_{0} \in D(A)$.

We mention that in many cases of interest $C$ is a non-closable operator, when regarded as a densely defined operator on $X$.

Definition 2.2. With the above notation, the $L$-extension of $C$ is the operator defined by

$$
C_{L} x_{0}=\lim _{\tau \rightarrow 0} C \frac{1}{\tau} \int_{0}^{\tau} \mathbb{T}_{\sigma} x_{0} d \sigma
$$

for all $x_{0}$ in the domain

$$
D\left(C_{L}\right)=\left\{x_{0} \in X: \text { the limit in (2.1) exists }\right\} .
$$

We define on $D\left(C_{L}\right)$ the norm

$$
\left\|x_{0}\right\|_{D\left(C_{L}\right)}=\left\|x_{0}\right\|_{X}+\sup _{r \in(0,1)}\left\|C \frac{1}{\tau} \int_{0}^{\tau} \mathbb{T}_{\sigma} x_{0} d \sigma\right\|_{Y} .
$$

Definition 2.3. With the notation of Definition 2.1, the $\Lambda$-extension of $C$ is the operator defined by

$$
C_{\Lambda} x_{0}=\lim _{\lambda \rightarrow+\infty} C \lambda(\lambda I-A)^{-1} x_{0}
$$

( $\lambda$ is real), for all $x_{0}$ in the domain

$$
D\left(C_{\Lambda}\right)=\left\{x_{0} \in X \mid \text { the limit in (2.2) exists }\right\} .
$$

Take $\lambda_{0} \in \mathbb{R}$ such that $\left[\lambda_{0}, \infty\right) \subset \rho(A)$. We define on $D\left(C_{\Lambda}\right)$ the norm

$$
\left\|x_{0}\right\|_{D\left(C_{\Lambda}\right)}=\left\|x_{0}\right\|_{X}+\sup _{\lambda \geq \lambda_{0}}\left\|C \lambda(\lambda I-A)^{-1} x_{0}\right\|_{Y} .
$$

It is not difficult to show (see [8-10]) that with their new norms, $D\left(C_{L}\right)$ and $D\left(C_{\Lambda}\right)$ are Banach spaces, and $C_{L}$ and $C_{\Lambda}$ are bounded operators from their respective domains to $Y$. It can also be shown that

$$
X_{1} \subset D\left(C_{\Lambda}\right) \subset D\left(C_{\Lambda}\right) \subset X
$$

with continuous embeddings. Moreover, $C_{\Lambda}$ is an extension of $C_{L}$, which is an extension of $C$. It is clear from the definition of admissibility that the operator $\Psi_{\tau}: D(A) \rightarrow$ $L^{2}([0, \tau], Y)$ defined by

$$
\left(\Psi_{\tau} x_{0}\right)(t)=C \mathbb{T}_{t} x_{0} \quad \text { for all } \quad t \in[0, \tau]
$$


has a continuous extension to $X$, which we denote by the same symbol. It is not difficult to show (using the concept of Lebesgue point of a function) that the operator $\psi_{\tau}$ can be represented as follows:

$$
\left(\Psi_{\tau} x_{0}\right)(t)=C_{L} \mathbb{T}_{t} x_{0}
$$

for every $x_{0} \in X$ and for almost every $t \in[0, \tau]$. We introduce the operator $\psi_{\infty}: X \rightarrow$ $L_{\text {loc }}^{2}([0, \infty), Y)$ by formula $(2.3)$, which makes sense for almost every $t \in[0, \infty)$. Then the Laplace transform of $\psi_{\infty} x_{0}$ is $C(s I-A)^{-1} x_{0}$.

Proposition 2.4. For any $x_{0} \in D\left(C_{L}\right)$ we have

$$
C_{L} x_{0}=\lim _{\lambda \rightarrow+\infty} C \lambda^{2}(\lambda I-A)^{-2} x_{0} .
$$

Proof. Consider the function $v(t)=e^{-\omega t} \Psi_{\infty} x_{0}$, which for $\omega$ large enough is in $L^{2}([0, \infty), Y)$. Its Laplace transform is $\hat{v}(s)=C((s+\omega) I-A)^{-1} x_{0}$. The fact that $x_{0} \in D\left(C_{L}\right)$ means that the limit on the left of (1.14) exists, and equals $C_{L} x_{0}$. By the Abelian theorem mentioned at the end of Section $1, s \hat{v}(s)$ has the same angular limit at $+\infty$. Now by Proposition 1.2 we have

$$
\lim _{\lambda \rightarrow+\infty} C \lambda^{2}((\lambda+\omega) I-A)^{-2} x_{0}=C_{L} x_{0}
$$

which is equivalent to the identity to be proved

Problem 2.5. Can the inclusion $D\left(C_{L}\right) \subset D\left(C_{\Lambda}\right)$ be strict?

This problem was raised in [9]. Using the results from Section 1, we shall give an affirmative answer to it in Example 2.7. Here is another, related problem raised in [10] (with a prize) which is still open:

Problem 2.6. Is $D(A)$ dense in the Banach space $D\left(C_{\Lambda}\right)$ ?

It is worth mentioning here that any $x_{0} \in D\left(C_{\Lambda}\right)$ for which the identity

$$
C_{\Lambda} x_{0}=\lim _{\lambda \rightarrow+\infty} C \lambda^{2}(\lambda I-A)^{-2} x_{0}
$$

holds (in particular, any $x_{0} \in D\left(C_{L}\right)$ ) can be approximated by elements of $D(A)$ as follows: $x_{0}=\lim _{\lambda \rightarrow+\infty} \lambda(\lambda I-A)^{-1} x_{0}$, the convergence being in the norm of $D\left(C_{\Lambda}\right)$. A related fact, proved in [10], is that $D(A)$ is dense in $D\left(C_{L}\right)$.

Example 2.7. We take $X=L^{2}[0, \infty)$ and $\mathbb{T}$ is the left shift semigroup on $X$, i.e., $\left(\mathbb{T}_{t} x\right)(\xi)=x(\xi+t)$. The generator of this semigroup is $A=\frac{d}{d \xi}$, with domain $D(A)=H^{1}[0, \infty)$. We take $Y=I C$ and define $C: D(A) \longrightarrow Y$ by $C x=x(0)$. Then it is easy to see that the operator $\Psi_{\infty}$ is given by $\left(\Psi_{\infty} x\right)(t)=x(t)$, whence $\left(C(s I-A)^{-1} x\right)(s)=\hat{x}(s)$. Now it is clear that $D\left(C_{L}\right)$ consists of those $v \in L^{2}[0, \infty)$ for which the limit on the left of (1.14) exists, while $D\left(C_{\Lambda}\right)$ consists of those $v$ for which the limit on the right of (1.14) exists. By Proposition 1.5, the inclusion $D\left(C_{L}\right) \subset D\left(C_{\Lambda}\right)$ is strict.

Now we recall very briefly some facts about regular linear systems, so that the reader can get an idea from where the problems discussed earlier arose. An abstract 
linear system is a linear time-invariant system such that, on any finite time interval, the operator from the initial state and the input function to the final state and the output function is bounded. The input, state and output spaces are Hilbert spaces and the input and output functions are of class $L_{\mathrm{loc}}^{2}$. For the detailed definition we refer to Salamon [6], or to Weiss [9]. The input to output operator of any abstract linear system can be described by a transfer function, which is an analytic operator-valued function defined on some right half-plane in $\mathbb{C}$. The transfer function of any abstract linear system is well posed, meaning that it is bounded on some right half-plane (not necessarily the same where it is defined). We do not distinguish between two transfer functions defined on two different right half-planes, if one function is a restriction of the other (thus, we are in fact dealing with equivalence classes of analytic functions).

Let $\Sigma$ be an abstract linear system, with input space $U$, state space $X$ and output space $Y$. Let $\mathbb{T}$ be the semigroup of $\Sigma$, i.e., the strongly continuous semigroup on $X$ which describes the evolution of the state of $\Sigma$ if the input function is zero. Let $A$ denote the generator of $\mathbb{T}$. The Hilbert space $X_{-1}$ is defined as the completion of $X$ with respect to the norm $\|x\|_{-1}=\left\|(\beta I-A)^{-1} x\right\|$, where $\beta \in \rho(A)$ is fixed. The space $X_{1}$ was introduced earlier. We have

$$
X_{1} \subset X \subset X_{-1}
$$

and the semigroup $\mathbb{T}$ can be extended to a semigroup on $X_{-1}$. These two semigroups are isomorphic and we shall denote them by the same symbol. The generator of $\mathbb{T}$ on $X_{-1}$ is an extension of $A$ to $X$, also denoted $A$.

The state of $\Sigma$ at any moment $\tau \geq 0$ can be expressed by the formula

$$
x(\tau)=\mathbb{T}_{\tau} x(0)+\int_{0}^{\tau} \mathbb{T}_{\tau-\sigma} B u(\sigma) d \sigma
$$

Here $u \in L_{\text {loc }}^{2}([0, \infty), U)$ is the input function and $B \in \mathcal{L}\left(U, X_{-1}\right)$ is the control operator of $\Sigma$. We have $x(\tau) \in X$ and $x(\tau)$ depends continuously on $\tau$, on $x(0)$ and on $u$. The transfer function from the input to the state is $(s I-A)^{-1} B$.

If $u=0$ and $x(0) \in X_{1}$, then the output function of $\Sigma$ on $[0, \infty)$ is

$$
y(t)=C T_{t} x(0) .
$$

Here $C \in \mathcal{L}\left(X_{1}, Y\right)$ is the observation operator of $\Sigma$. It is an admissible observation operator for $\mathbb{T}$, as defined earlier.

Let $\mathbf{G}$ denote the transfer function of $\Sigma$. It is called regular if the limit

$$
D v=\lim _{\lambda \rightarrow+\infty} \mathbf{G}(\lambda) v
$$

exists for every $v \in U$ ( $\lambda$ is real). Then $D \in \mathcal{L}(U, Y)$ is called the feedthrough operator of $\mathbf{G}$. If $\mathbf{G}$ is regular, then $\Sigma$ is called a regular linear system, abreviated. The regularity condition (2.4) can be formulated in many different ways, of which we mention the following: $\Sigma$ is regular if and only if the product $C_{L}(s I-A)^{-1} B$ makes sense for some (hence for any) $s \in \rho(A)$, i.e.,

$$
\operatorname{Ran}(s I-A)^{-1} B \subset D\left(C_{L}\right) .
$$


The proof of this fact is based on Theorem 1.4. In the above criterion, $C_{L}$ may be replaced with $C_{\Lambda}$. Another equivalent condition to regularity is that any step response of $\Sigma$ has, a Cesàro limit at $t=0$.

If $\Sigma$ is regular, then

$$
\mathbf{G}(s)=C_{L}(s I-\dot{A})^{-1} B+D
$$

for any $s \in C$ with Re $s$ sufficiently large. Moreover, in the time domain, $\Sigma$ is completely described by the equations

$$
\begin{aligned}
& \dot{x}(t)=A x(t)+B u(t) \\
& y(t)=C_{L} x(t)+D u(t)
\end{aligned}
$$

which hold for almost every $t \geq 0$ (in particular, $x(t) \in D\left(C_{L}\right)$ for a.e. $t \geq 0$ ). $x(\cdot$ ) is the unique strong solution (in $X_{-1}$ ) of equation $(2.6)_{a}$, for any given $x(0) \in X$ and $u \in L_{\text {loc }}^{2}([0, \infty), U)$. The output function belongs to $L_{\text {loc }}^{2}([0, \infty), Y)$.

The operators $A, B, C$ and $D$ appearing in (2.6) are called the generating operators of $\Sigma$, or its generators for short.

Now we turn to feedback, following [10]. Let $U$ and $Y$ be Hilbert spaces, suppose $\mathbf{G}$ is an $\mathcal{L}(U, Y)$-valued well posed transfer function and let $K \in \mathcal{L}(Y, U) . \quad K$ is an admissible feedback operator for $\mathbf{G}$ if $I-K \mathbf{G}$ is invertible on some right half-plane and its inverse is a well posed transfer function (equivalently, if $I-G K$ has the same property). Then the function $\mathbf{G}^{K}$ defined by

$$
\mathbf{G}^{K}(s)=\mathbf{G}(s)(I-K \mathbf{G}(s))^{-1}
$$

is called the closed loop transfer function corresponding to $\mathbf{G}$ and $K$.

Now suppose $\mathbf{G}$ is regular and let $D$ be its feedthrough operator. If $K$ is an admissible feedback operator for $\mathbf{G}$, then $\mathbf{G}^{K}$ (given by (2.7)) is regular if and only if $I-D K$ is invertible (equivalently, if and only if $I-K D$ is invertible). If $\mathbf{G}^{K}$ is regular, then its feedthrough operator is

$$
D^{K}=(I-D K)^{-1} D=D(I-K D)^{-1} \text {. }
$$

Under certain additional assumptions (e.g., if at least one of the spaces $U$ and $Y$ is finite-dimensional), the invertibility of $I-D K$ follows from the admissibility of $K$.

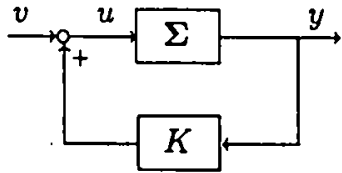

Figure 1. The closed loop system $\Sigma^{K}$

Let $\Sigma$ be an abstract linear system with transfer function $\mathbf{G}$. If $K$ is an admissible feedback operator for $G$, then there exists a unique closed loop system $\Sigma^{K}$ corresponding 
to $\Sigma$ and $K$, represented in Figure $1 . \Sigma^{K}$ is an abstract linear system and its transfer function is $\mathbf{G}^{K}$ from (2.7). For the precise definition of $\Sigma^{K}$ we refer to [10].

Now suppose $\Sigma$ is a regular linear system with generators $A, B, C$ and $D$, and $K$ and $\Sigma^{K}$ are as before. If $I-D K$ is invertible, then (as mentioned earlier) $\Sigma^{K}$ is regular and we can compute its generators via formulas which are similar to those for finitedimensional systems. Let $A^{K}, B^{K}, C^{K}, D^{K}$ be the generators of $\Sigma^{K}$. As we already know, $D^{K}$ is given by (2.8). The formula for $A^{K}$ is

$$
A^{K} x=\left(A+B K(I-D K)^{-1} C_{L}\right) x
$$

defined for all $x$ in the domain

$$
D\left(A^{K}\right)=\left\{x \in D\left(C_{L}\right) B i g \mid\left(A+B K(I-D K)^{-1} C_{L}\right) x \in X\right\}
$$

The operators $C^{K}$ and $B^{K}$ are given by

$$
\begin{aligned}
C^{K} x & =(I-D K)^{-1} C_{L} x \quad \text { for all } x \in D\left(A^{K}\right) \\
B^{K} & =B(I-K D)^{-1} .
\end{aligned}
$$

It is clear that in (2.9) - (2.11) $C_{L}$ can be replaced by its extension $C_{\Lambda}$. To understand (2.12) better, it should be pointed out that $B^{K} \in \mathcal{L}\left(u, X_{-1}^{K}\right)$, where $X_{-1}^{K}$ is the analogue of $X_{-1}$ for $\Sigma^{K}$, but in fact both $B$ and $B^{K}$ are in $\mathcal{L}(U, W)$, where $W$ is a Banach space such that $W \subset X_{-1} \cap X_{-1}^{K}$. More precisely, $W=(\beta I-A) W_{1}$, where $W_{1}$ is the closure of $D(A)$ in the Banach space $D\left(C_{\Lambda}\right)$. If Problem 2.6 would have an affirmative solution, then we would have $W_{1}=D\left(C_{\Lambda}\right)$ and the representation of $B^{K}$ would simplify. It is interesting to mention that both $W_{1}$ and $W$ are invariant under feedback, i.e., if we compute them for the closed loop system $\Sigma^{K}$, we get the same spaces. For more details see Section 7 of $[10]$.

The $\Lambda$-extensions $C_{\Lambda}$ and $C_{\Lambda}^{K}$ are related by

$$
D\left(C_{\Lambda}^{K}\right)=D\left(C_{\Lambda}\right) \quad \text { and } \quad C_{\Lambda}^{K}=(I-D K)^{-1} C_{\Lambda} .
$$

Unfortunately, we do not know of any such simple relationship between the $L$-extensions of $C$ and $C^{K}$. It is formula (2.13) which makes the use of $C_{\Lambda}$ sometimes more convenient compared to $C_{L}$.

Both $A$ and $A^{K}$ can be extended to bounded operators from $W_{1}$ to $W$ and formula (2.9) remains valid for $A^{K}$ on $W_{1}$, with the minor adjustment that $C_{L}$ has to be replaced by its extension $C_{\Lambda}$. 


\section{The idea of the proof: a special Blaschke product}

The proof of Theorem 1.1 (the construction of the counterexample) is rather involved and so we devide it into three sections, this being the first of them. Here we give an outline of the proof.

The core of the proof of is a special Blaschke product $B_{p}$ on $\mathbb{C}_{+}$, which depends on the parameter $p$ appearing in Theorem 1.1. We shall construct $B_{p}$ by specifying the sequence $\left\{a_{k}\right\}$ of its zeros, which are all positive: $0<a_{1}<a_{2}<a_{3}<\ldots$. Then for any $s \in \mathbb{C}_{+}$

$$
B_{p}(s)=\prod_{1 \leq k<\infty} \frac{a_{k}-s}{a_{k}+s} .
$$

We shall specify the sequence $\left\{a_{k}\right\}$ in Section 4 . Now we only list the properties of $\left\{a_{k}\right\}$ and of $B_{p}$ which we need and we show how, if those properties are satisfied, the functions $f_{p}$ of Theorem 1.1 can be constructed from $B_{p}$. We denote

$$
\omega=\frac{1}{\pi}\left(1-\frac{1}{p}\right)^{\frac{1}{2}}
$$

The sequence $\left\{a_{k}\right\}$ should have the following properties:

(A1) The "rough asymptotics" of $\left(a_{k}\right)$ is

$$
a_{k}=e^{\sqrt{k} / \omega}(1+o(1)) \quad(k \rightarrow \infty)
$$

(A2) $\left\{a_{k}\right\}$ satisfies the "separation property"

$$
\frac{a_{k+1}-a_{k}}{a_{k+1}}=\frac{1}{2 \omega \sqrt{k}}(1+o(1)) \quad(k \rightarrow \infty) .
$$

The Blaschke product $B_{p}$ should have the following properties:

(UE) (Upper Estimate) There exists a $C>0$ such that for all $x \in(0, \infty)$

$$
\left|B_{p}(x)\right| \leq C(1+x)^{-(1-1 / p)} .
$$

(LE) (Lower Estimate) For any $\varepsilon \in\left(0, \frac{1}{2}\right)$ we define a neighbourhood $N(\varepsilon)$ of the set $\left\{a_{k} \mid k \in \mathbb{N}\right\}$ by

$$
N(\varepsilon)=\bigcup_{1 \leq k<\infty} N\left(\varepsilon, a_{k}\right)
$$

where

$$
N\left(\varepsilon, a_{k}\right)=\left(a_{k}-\varepsilon\left(a_{k}-a_{k-1}\right), a_{k}+\varepsilon\left(a_{k+1}-a_{k}\right)\right)
$$

We require that for some (fixed) $\varepsilon \in\left(0, \frac{1}{2}\right)$ there should exist a $c(\varepsilon)>0$ such that

$$
\left|B_{p}(x)\right| \geq c(\varepsilon)(1+x)^{-(1-1 / p)} \quad \text { for all } x \in(0, \infty) \backslash N(\varepsilon) .
$$


We explain how a Blaschke product $B_{p}$ satisfying the above requirements enables us to construct $f_{p}$ for Theorem 1.1. We define $f_{p}$ on $\mathbb{C}_{+}$by

$$
f_{p}(s)=\frac{1}{(s+1)^{1 / p}} \cdot \frac{1}{[\ln (s+2)]^{1 / p}} \cdot \frac{1}{[\ln \ln (s+3)]^{2 / p}} B_{p}(s)
$$

(branches of the multivalued functions are chosen is such a way that $f_{p}$ takes real values on $(0, \infty))$. Since $\left|B_{p}(i y)\right|=1$ for real $y$, we see that $f_{p} \in H^{\infty}$ and

$$
\left|f_{p}(i y)\right|^{p}=\frac{1}{|y| \ln |y|(\ln \ln |y|)^{2}}(1+o(1)) \quad(y \rightarrow \pm \infty) .
$$

This shows that $f_{p} \in H^{p}$. Since for any $q \geq p$ we have $H^{p} \cap H^{\infty} \subset H^{q}$, we have shown that $f_{p}$ satisfies (i) of Theorem 1.1.

From (3.1) and (UE) we see that for all $x>0$

$$
f_{p}(x) \leq C \frac{1}{|x+1|} \cdot \frac{1}{[\ln (x+2)]^{1 / p}} \cdot \frac{1}{[\ln \ln (x+3)]^{2 / p}}
$$

which implies (ii) of Theorem 1.1. Condition (iii) is clearly satisfied.

It remains to show that (iv) of Theorem 1.1 holds. For this, we need a lower estimate of $\left|f_{p}^{\prime}\right|$ in certain points. Since $f_{p}\left(a_{k}\right)=f_{p}\left(a_{k+1}\right)=0$,

$$
\max _{x \in\left[a_{k}, a_{k+1}\right]}\left|f_{p}^{\prime}(x)\right| \geq \frac{2}{a_{k+1}-a_{k}} \cdot \max _{x \in\left[a_{k}, a_{k+1}\right]}\left|f_{p}(x)\right|
$$

In particular, denoting by $b_{k}$ a point in $\left[a_{k}, a_{k+1}\right]$ where the maximum of $\left|f_{p}^{\prime}\right|$ (in that interval) is attained, we have

$$
\left|f_{p}^{\prime}\left(b_{k}\right)\right| \geq \frac{2}{a_{k+1}-a_{k}}\left|f_{p}\left(\frac{a_{k}+a_{k+1}}{2}\right)\right| .
$$

From properties (A1) and (A2) we derive that

$$
\frac{1}{a_{k+1}-a_{k}}=\frac{2 \omega^{2} \ln a_{k+1}}{a_{k+1}}(1+o(1)) \quad(k \rightarrow \infty)
$$

In order to estimate $\left|f_{p}\left(\frac{a_{k}+a_{k+1}}{2}\right)\right|$ from below, we use the fact that $\frac{a_{k}+a_{k+1}}{2}$ is certainly not in $N(\varepsilon)$, so that by property (LE)

$$
\left|B_{p}\left(\frac{a_{k}+a_{k+1}}{2}\right)\right| \geq c(\varepsilon)\left[\frac{a_{k}+a_{k+1}}{2}\right]^{-(1-1 / p)}>c(\varepsilon) \frac{1}{\left[a_{k+1}+1\right]^{1-1 / p}} .
$$

Hence by (3.1)

$$
\left|f_{p}\left(\frac{a_{k}+a_{k+1}}{2}\right)\right| \geq c(\varepsilon) \cdot \frac{1}{a_{k+1}+1} \cdot \frac{1}{\left[\ln \left(a_{k+1}+2\right)\right]^{1 / p}} \cdot \frac{1}{\left[\ln \ln \left(a_{k+1}+3\right)\right]^{2 / p}} .
$$


Substituting this and (3.3) into (3.2), we obtain that for large $k$

$$
\left|f_{p}^{\prime}\left(b_{k}\right)\right| \geq c_{1}(\varepsilon) \frac{1}{a_{k}^{2}} \frac{\left(\ln a_{k}\right)^{1-1 / p}}{\left(\ln \ln a_{k}\right)^{2 / p}}
$$

where $c_{1}(\varepsilon)$ does not depend on $k$. From here, for large $k$,

$$
b_{k}^{2}\left|f_{p}^{\prime}\left(b_{k}\right)\right| \geq c_{1}(\varepsilon) \frac{\left(\ln a_{k}\right)^{1-1 / p}}{\left(\ln \ln a_{k}\right)^{2 / p}} \longrightarrow \infty .
$$

Since the sign of $f_{p}$ changes at each zero, (iv) of Theorem 1.1 holds as well.

Numerical tests. For reasons which will be explained in Section 4, our choice of $a_{k}$ is

$$
a_{k}=2 \omega^{2} \int_{\exp (\sqrt{k-1} / \omega)}^{\exp (\sqrt{k} / \omega)} \ln \lambda d \lambda
$$

(which can be computed easily). Using MATLAB, we have computed approximately the Blaschke product $B_{2}(x)$ by multiplying the first 500 factors. From here, we have computed $x f_{2}(x)$ for $x \in\left(10^{15}, 10^{18}\right)$ and we have plotted the results in Figure 2 . On the horizontal axis we have $\log _{2} x$, which is better suited for visualization than $x$. The zeros $a_{k}$ with '61 $\leq k \leq 87$ are visible in the graph. We can see that $x f_{2}(x)$ converges slowly to zero.

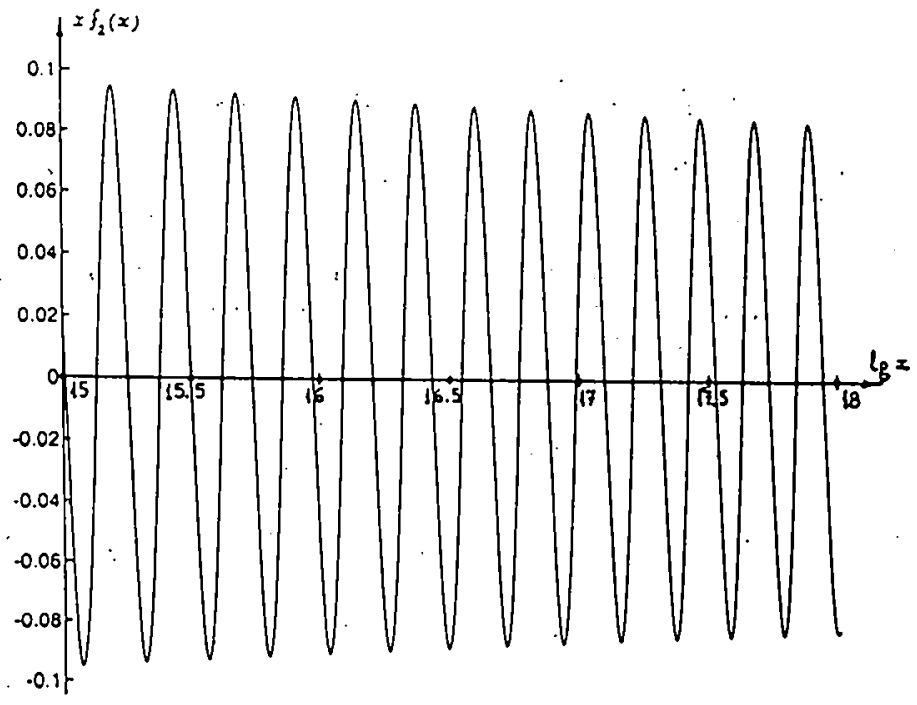

Figure 2: The function $x f_{2}(x)$ for $x$ between $10^{15}$ and $10^{18}$

To test the lower estimate (LE), we have computed $\left|B_{2}\left(m_{k}\right)\right| \sqrt{1+m_{k}}$ for $1 \leq k \leq$ 100 , where $m_{k}=\frac{1}{2}\left(a_{k}+a_{k+1}\right)$. The results are plotted in Figure 3, where we see that 
$\left|B_{2}\left(m_{k}\right)\right| \sqrt{1+m_{k}}$ is slowly increasing, in particular, it must be bounded from below (in Figure 3 , the discrete set of values has been interpolated to form a continuous curve).

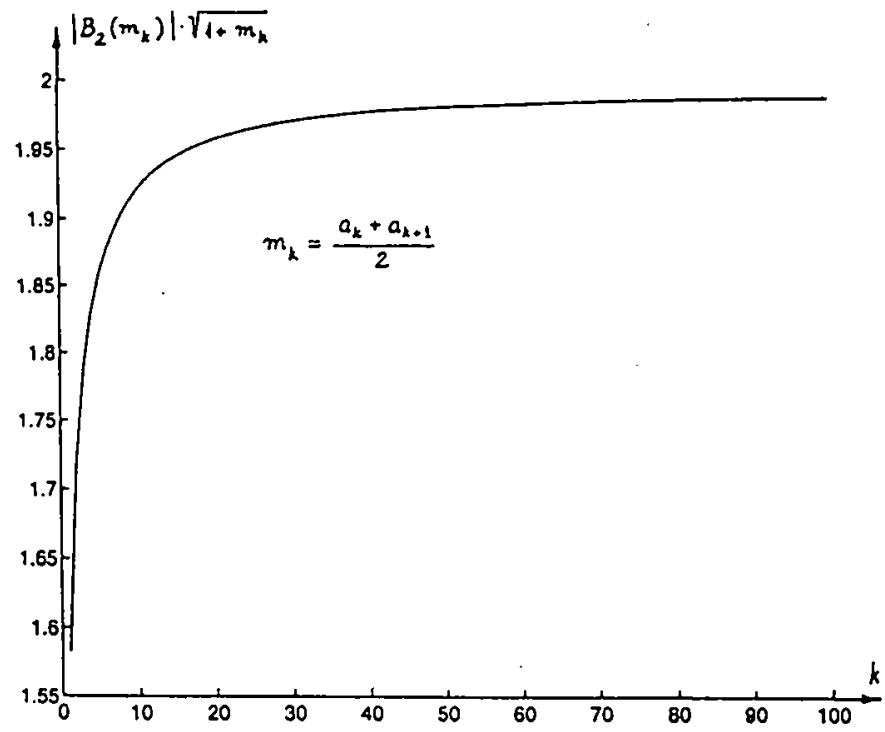

Figure 3: The function $\left|B_{2}\left(m_{k}\right)\right| \sqrt{1+m_{k}}$ for $k$ between 1 and 100

Acknowledgment. We are indebted to Hao Zhong, who studies at Ben-Gurion University, for writing the programs needed to create Figures 2 and 3.

\section{The comparison potential and atomization}

In this section we shall define the sequence $\left\{a_{k}\right\}$ which determines $B_{p}$ and we shall verify that it satisfies the conditions (A1) and (A2) from Section 3.

The requirements on the Blaschke product $B_{p}$ listed in Section 3 are such that the argument of $B_{p}$ is not important for us, only its absolute value. Thus we may look at the subharmonic function defined on $\mathbb{C}_{+}$by

$$
\ln \left|B_{p}(s)\right|=\sum_{k=1}^{\infty} \ln \left|\frac{a_{k}-s}{a_{k}+s}\right|
$$

and try to define $\left\{a_{k}\right\}$ in such a way that for all $x>0$ (by property (UE))

$$
\ln \left|B_{p}(x)\right| \leq-\left(1-\frac{1}{p}\right) \ln (1+x)+\ln C
$$

and for all $x \in(0, \infty) \backslash N(\varepsilon)$ (by property (LE))

$$
\ln \left|B_{p}(x)\right| \geq-\left(1-\frac{1}{p}\right) \ln (1+x)+\ln c(\varepsilon) .
$$


The Riesz measure of the subharmonic function $\ln \left|B_{p}\right|$ is discrete and concentrated on the set $\left\{a_{k} \mid k \in \mathbb{N}\right\}$. We shall define a continuous measure whose potential satisfies the desired estimates on $(0, \infty)$. Then we shall construct the sequence $\left\{a_{k}\right\}$ by an "atomization procedure" of this continuous measure.

Define for all $\lambda>0$

$$
n(\lambda)=\omega^{2} \ln ^{2} \lambda
$$

where $\pi^{2} \omega^{2}=1-\frac{1}{p}$, as in Section 3. We also define for all $s \in C_{+}$

$$
V(s)=\int_{0}^{+\infty} \ln \left|\frac{\lambda-s}{\lambda+s}\right| \operatorname{dn}(\lambda)
$$

Luckily, $V$ can be calculated explicitly. ( $V$ is not subharmonic in $\mathbb{C}_{+}$, because $n$ decreases on the interval $(0,1)$, but this is not important.) Integrating by parts in (4.4) we obtain that for $x>0$

$$
V(x)=-\mathrm{P} . \mathrm{V} \cdot \int_{0}^{+\infty}\left(\frac{1}{\lambda-x}-\frac{1}{\lambda+x}\right) n(\lambda) d \lambda
$$

where P.V. is the so-called principle value of the integral. Substituting (4.3) into the last formula, we get

$$
V(x)=-2 \omega^{2} \text { P.V. } \int_{0}^{+\infty} \frac{x}{\lambda^{2}-x^{2}} \ln ^{2} \lambda d \lambda .
$$

By the change of variables $\lambda \rightarrow x \lambda$ we obtain

$$
V(x)=-2 \omega^{2} \text { P.V. } \int_{0}^{+\infty} \frac{1}{\lambda^{2}-1}(\ln \lambda+\ln x)^{2} d \lambda .
$$

We decompose $(\ln \lambda+\ln x)^{2}$ into the usual sum of three terms and notice that two of them do not contribute to the integral (use the change of variable $\lambda \rightarrow 1 / \lambda$ to check this):

$$
\int_{0}^{+\infty} \frac{\ln ^{2} \lambda}{\lambda^{2}-1} d \lambda=0 \quad \text { and } \quad \text { P.V. } \int_{0}^{+\infty} \frac{1}{\lambda^{2}-1} d \lambda=0 .
$$

Thus, for all $x>0$,

$$
V(x)=-(\ln x) 4 \omega^{2} \int_{0}^{\infty} \frac{\ln \lambda}{\lambda^{2}-1} d \lambda .
$$

This integral can be calculated explicitly:

$$
2 \int_{0}^{+\infty} \frac{\ln \lambda}{\lambda^{2}-1} d \lambda=\int_{-\infty}^{+\infty} \frac{\ln |\lambda|}{\lambda^{2}-1} d \lambda=\operatorname{Re}\left(\text { P.V. } \int_{-\infty}^{+\infty} \frac{\ln \lambda}{\lambda^{2}-1} d \lambda\right)
$$


(where $\arg \lambda=\pi$ for $\lambda<0$ ). The last integral can be computed by means of residues:

$$
\text { P.V. } \int_{-\infty}^{+\infty} \frac{\ln \lambda}{\lambda^{2}-1} d \lambda=\left.\pi i \operatorname{Res}\right|_{\lambda=-1} \frac{\ln \lambda}{\lambda^{2}-1}=\pi i \frac{\ln e^{i \pi}}{2 e^{i \pi}}=\frac{\pi^{2}}{2}
$$

Thus we have obtained

$$
V(x)=-\pi^{2} \omega^{2} \ln x=-\left(1-\frac{1}{p}\right) \ln x .
$$

We shall obtain the sequence $\left\{a_{k}\right\}$ of zeros of $B_{p}$ by means of an "atomization" of the measure $d n$ generated by the function $n$ from (4.3). This atomization is the most delicate part of our construction. We obtain the estimates (4.1) and (4.2) for $\ln \left|B_{p}\right|$ by comparing the function $\ln \left|B_{p}\right|$, with the function $V$ which has been calculated explicitly in (4.5).

Denote

$$
\lambda_{k}=\exp \left\{\frac{1}{\omega} \sqrt{k}\right\} \quad\left(k \in N_{0}\right) .
$$

In other words, $\lambda_{k}$ is the solution of $n(\lambda)=k$. Clearly

$$
\int_{\lambda_{k-1}}^{\lambda_{k}} d n(\lambda)=1
$$

i.e., $d n$ is a probability measure on $\left[\lambda_{k-1}, \lambda_{k}\right]$. From (4.4) it follows that

$$
V(x)=\sum_{k=1}^{\infty} V_{k}(x)+R(x)
$$

where $R$ is defined by

$$
R(x)=\int_{0}^{1} \ln \left|\frac{\lambda-x}{\lambda+x}\right| \operatorname{dn}(\lambda)
$$

and

$$
V_{k}(x)=\int_{\lambda_{k-1}}^{\lambda_{k}} \ln \left|\frac{\lambda-x}{\lambda+x}\right| \operatorname{dn}(\lambda)
$$

Clearly

$$
\lim _{x \rightarrow+\infty} R(x)=0 .
$$

Let $a_{k}$ be the gravity center of the probability measure $d n$ on $\left[\lambda_{k-1}, \lambda_{k}\right]$ :

$$
a_{k}=\int_{\lambda_{k-1}}^{\lambda_{k}} \lambda d n(\lambda) .
$$


Let us prove that $\left\{a_{k}\right\}$ satisfies the conditions (A1) and (A2). First we estimate the length of the interval $\left[\lambda_{k-1}, \lambda_{k}\right]$. By (4.6)

$$
\lambda_{k}-\lambda_{k-1}=\exp \left\{\frac{1}{\omega} \sqrt{k}\right\}\left[1-\exp \left\{\frac{1}{\omega}(\sqrt{k-1}-\sqrt{k})\right\}\right] .
$$

Since $\sqrt{k-1}-\sqrt{k}=-\frac{1}{2 \sqrt{k}}(1+o(1))(k \rightarrow \infty)$ and $1-e^{-z}=z(1+o(z))$ for $|z| \rightarrow 0$,

$$
\lambda_{k}-\lambda_{k-1}=\frac{1}{2 \omega \sqrt{k}} \lambda_{k}(1+o(1)) \quad(k \rightarrow \infty),
$$

or

$$
\lambda_{k}-\lambda_{k-1}=\frac{\lambda_{k}}{2 \omega^{2} \ln \lambda_{k}}(1+o(1)) \quad(k \rightarrow \infty) .
$$

From (4.11) it follows that

$$
\lambda_{k-1}<a_{k}<\lambda_{k} .
$$

The "rough" asymptotics (A1) of the sequence $\left\{a_{k}\right\}$ follows from (4.15), (4.14) and (4.6). In order to obtain "the separation condition" (A2) we have to argue more accurately. From (4.3) and (4.11)

$$
a_{k}=2 \omega^{2} \int_{\lambda_{k-1}}^{\lambda_{k}} \ln \lambda d \lambda .
$$

This implies

$$
2 \omega^{2}\left(\ln \lambda_{k-1}\right)\left(\lambda_{k}-\lambda_{k-1}\right)<a_{k}<2 \omega^{2}\left(\ln \lambda_{k}\right)\left(\lambda_{k}-\lambda_{k-1}\right) .
$$

The above estimate determines a much shorter interval of possible values of $a_{k}$ than (4.15), asymptotically shorter by a factor of $\sqrt{k} / \omega$, as one can check by a simple computation using (4.14).

We want to get a more precise estimate for $\lambda_{k}-\lambda_{k-1}$, using (4.12). Since

$$
\sqrt{k-1}-\sqrt{k}=-k^{1 / 2}\left[1-\left(1-\frac{1}{k}\right)^{1 / 2}\right]=-k^{1 / 2}\left[1-\left(1-\frac{1}{2 k}+O\left(k^{-2}\right)\right)\right]
$$

we have

$$
\sqrt{k-1}-\sqrt{k}=-\frac{1}{2} k^{-1 / 2}+O\left(k^{-3 / 2}\right) \quad(k \rightarrow \infty) .
$$

Hence

$$
\exp \left\{\frac{1}{\omega}(\sqrt{k-1}-\sqrt{k})\right\}=1-\frac{1}{2 \omega} k^{-1 / 2}+\frac{1}{8 \omega^{2}} k^{-1}+O\left(k^{-3 / 2}\right)
$$

whence, by (4.6) and (4.12), we get that for $k \rightarrow \infty$

$$
\lambda_{k}-\lambda_{k-1}=\lambda_{k}\left[\frac{1}{2 \omega} k^{-1 / 2}-\frac{1}{8 \omega^{2}} k^{-1}+O\left(k^{-3 / 2}\right)\right] .
$$


Since $\ln \lambda_{k}=\frac{1}{\omega} k^{1 / 2}(\operatorname{see}(4.6))$, for $k \rightarrow \infty$ we have

$$
2 \omega^{2}\left(\ln \lambda_{k}\right)\left(\lambda_{k}-\lambda_{k-1}\right)=\lambda_{k}\left[1-\frac{1}{4 \omega} k^{-1 / 2}+O\left(k^{-1}\right)\right] .
$$

On the other hand,

$$
\ln \lambda_{k-1}=\frac{1}{\omega}(k-1)^{1 / 2}=\frac{1}{\omega} k^{1 / 2}\left(1-\frac{1}{k}\right)^{1 / 2}
$$

or

$$
\ln \lambda_{k-1}=\frac{1}{\omega} k^{1 / 2}\left[\left(1-\frac{1}{2 k}\right)+O\left(k^{-2}\right)\right] \quad(k \rightarrow \infty) .
$$

From here and from (4.17) it follows that for $k \rightarrow \infty$

$$
2 \omega^{2}\left(\ln \lambda_{k-1}\right)\left(\lambda_{k}-\lambda_{k-1}\right)=\lambda_{k}\left[1-\frac{1}{4 \omega} k^{-1 / 2}+O\left(k^{-1}\right)\right]
$$

From this, together with (4.16) and (4.18) we obtain the asymptotic equality

$$
a_{k}=\lambda_{k}\left(1-\frac{1}{4 \omega} k^{-1 / 2}+O\left(k^{-1}\right)\right) \quad(k \rightarrow \infty) .
$$

This implies

$$
a_{k}-a_{k-1}=\left(\lambda_{k}-\lambda_{k-1}\right)\left(1-\frac{1}{4 \omega} k^{-1 / 2}\right)+\lambda_{k-1} O\left(k^{-1}\right)
$$

Finally, from this and (4.13) we obtain (A2).

We remark that from (4.19) and (4.17)

$$
\frac{\lambda_{k}-a_{k}}{\lambda_{k}-\lambda_{k-1}}=\frac{1}{2}+O\left(k^{-1 / 2}\right) \quad(k \rightarrow \infty)
$$

In other words, $a_{k}$ lies asymptotically at the center of the interval $\left[\lambda_{k-1}, \lambda_{k}\right]$. 


\section{The upper and lower estimates of the Blaschke product}

Let $B_{p}$ be the Blaschke product constructed from the sequence $\left\{a_{k}\right\}$ of zeros defined in (4.11). We shall obtain the estimates (4.1) and (4.2) (equivalent to (UE) and (LE)) by comparing the function $\ln \left|B_{p}\right|$ with the function $V$ defined in (4.4). We represent these functions as series and then compare these term by term.

We denote

$$
b_{k}(s)=\frac{a_{k}-s}{a_{k}+s}
$$

Clearly,

$$
\ln \left|B_{p}(s)\right|=\sum_{k=1}^{\infty} \ln \left|b_{k}(s)\right|
$$

In view of (4.7),

$$
\ln \left|b_{k}(s)\right|=\int_{\lambda_{k-1}}^{\lambda_{k}} \ln \left|\frac{a_{k}-s}{a_{k}+s}\right| d n(\lambda) .
$$

From here, with (4.9), it follows that for all $k \in \mathbb{N}$ and all $x>0$

$$
V_{k}(x)-\ln \left|b_{k}(x)\right|=\int_{\lambda_{k-1}}^{\lambda_{k}} \ln \left|\frac{\lambda-x}{a_{k}-x}\right| \operatorname{dn}(\lambda)-\int_{\lambda_{k-1}}^{\lambda_{k}} \ln \left|\frac{\lambda+x}{a_{k}+x}\right| d n(\lambda) .
$$

From (5.1) and (4.8) we obtain that for all $x>0$

$$
\ln \left|B_{p}(x)\right|-V(x)=\sum_{k=1}^{\infty}\left[\ln \left|b_{k}(x)\right|-V_{k}(x)\right]-R(x)
$$

In view of (4.10) we only have to estimate the infinite sum in (5.4).

Lemma. For any $\delta>0$ there is a $C(\delta)>0$ such that for any $t \in \mathbb{R}$

$$
|1+t| \geq \delta \Longrightarrow|\ln | 1+t\left|-t+\frac{t^{2}}{2}\right| \leq C(\delta)|t|^{3} .
$$

The proof of this lemma is left to the reader.

Let $x \in[1, \infty)$. Since $[1, \infty)=\bigcup_{1 \leq k<\infty}\left[\lambda_{k-1}, \lambda_{k}\right]$, there is an $m \in \mathbb{N}$ such that $x \in\left[\lambda_{m-1}, \lambda_{m}\right]$. We split the series in (5.4) into two sums as follows:

$$
\sum_{k=1}^{\infty}\left[\ln \left|b_{k}(x)\right|-V_{k}(x)\right]=\sum_{\substack{k \in \mathbb{N} \\|k-m|>1}} \cdots+\sum_{k=m-1}^{m+1} \ldots
$$

We shall estimate these sums term by term, using formula (5.3). We have grouped in the second sum the terms for which the variable $\lambda$ is close to $x$. To estimate the integrand in (5.3) we use the Lemma, taking into account that

$$
\frac{\lambda-x}{a_{k}-x}=1+\frac{\lambda-a_{k}}{a_{k}-x} \quad \text { and } \quad \frac{\lambda+x}{a_{k}+x}=1+\frac{\lambda-a_{k}}{a_{k}+x} .
$$


It is easy to see that

$$
\left|\frac{\lambda-x}{a_{k}-x}\right| \geq \delta>0 . \quad \text { for } \lambda \in\left[\lambda_{k-1}, \lambda_{k}\right], x \in\left[\lambda_{m-1}, \lambda_{m}\right] \text { and }|k-m|>1
$$

where $\delta$ does not depend on $k$ and $m$. It is also casy to see that

$$
\left|\frac{\lambda+x}{a_{k}+x}\right| \geq \delta>0 \quad \text { for } \lambda \in\left[a_{k-1}, a_{k}\right], x \in\left[a_{m-1}, a_{m}\right] \text { and all } k, m \geq 1
$$

with some positive $\delta$ not depending on $k$ and $m$. By the Lemma, with $t=\frac{\lambda-a_{k}}{a_{k}-x}$ and $t=\frac{\lambda+a_{k}}{a_{k}+x}$, and taking (4.7) into account, we obtain that for $|k-m|>1$

$$
\begin{gathered}
\left|\int_{\lambda_{k-1}}^{\lambda_{k}} \ln \right| \frac{\lambda-x}{a_{k}-x}\left|\ln (\lambda)-\int_{\lambda_{k-1}}^{\lambda_{k}} \frac{\lambda-a_{k}}{a_{k}-x} d n(\lambda)+\frac{1}{2} \int_{\lambda_{k-1}}^{\lambda_{k}} \frac{\left(\lambda-a_{k}\right)^{2}}{\left(a_{k}-x\right)^{2}} d n(\lambda)\right| \\
\leq C_{1} \max _{\lambda \in\left[\lambda_{k-1}, \lambda_{k}\right]} \frac{\left|\lambda-a_{k}\right|^{3}}{\left|a_{k}-x\right|^{3}}
\end{gathered}
$$

and for all $k, m \in \mathbb{N}$

$$
\begin{gathered}
\left|\int_{\lambda_{k-1}}^{\lambda_{k}} \ln \right| \frac{\lambda+x}{a_{k}+x}\left|\operatorname{dn}(\lambda)-\int_{\lambda_{k-1}}^{\lambda_{k}} \frac{\lambda-a_{k}}{a_{k}+x} d n(\lambda)+\frac{1}{2} \int_{\lambda_{k-1}}^{\lambda_{k}} \frac{\left(\lambda-a_{k}\right)^{2}}{\left(a_{k}+x\right)^{2}} d n(\lambda)\right| \\
\leq C_{1} \max _{\lambda \in\left[\lambda_{k-1}, \lambda_{k}\right]} \frac{\left|\lambda-a_{k}\right|^{3}}{\left|a_{k}+x\right|^{3}}
\end{gathered}
$$

where $C_{1}<\infty$ is a constant not depending on $\lambda, k$ and $x$. Clearly

$$
\max _{\lambda \in\left\{\lambda_{k-1}, \lambda_{k}\right]}\left|\lambda-a_{k}\right| \leq a_{k+1}-a_{k}
$$

In view of $(4.11)$, the integrals

$$
\int_{\lambda_{k-1}}^{\lambda_{k}} \frac{\lambda-a_{k}}{a_{k}-x} d n(\lambda) \quad \text { and } \quad \int_{\lambda_{k-1}}^{\lambda_{k}} \frac{\lambda-a_{k}}{a_{k}+x} d n(\lambda)
$$

vanish. (The points $a_{k}$ have been chosen in such a way that these integrals vanish.) Thus, from the above inequalities it follows that for $|k-m|>1$.

$$
\begin{aligned}
& \quad \int_{\lambda_{k-1}}^{\lambda_{k}}\left(\ln \left|\frac{\lambda-x}{a_{k}-x}\right|-\ln \left|\frac{\lambda+x}{a_{k}+x}\right|\right) d n(\lambda) \mid \\
& \quad \leq \frac{1}{2}\left(\frac{1}{\left(a_{k}-x\right)^{2}}-\frac{1}{\left(a_{k}+x\right)^{2}}\right) \int_{\lambda_{k-1}}^{\lambda_{k}}\left(\lambda-a_{k}\right)^{2} d n(\lambda)+2 C_{1} \frac{\left(a_{k+1}-a_{k}\right)^{3}}{\left(a_{k}-x\right)^{3}} .
\end{aligned}
$$


Since .

and

$$
\frac{1}{2}\left(\frac{1}{\left(a_{k}-x\right)^{2}}-\frac{1}{\left(a_{k}+x\right)^{2}}\right)=\frac{2 a_{k} x}{\left(a_{k}-x\right)^{2}\left(a_{k}+x\right)^{2}}
$$

$$
\int_{\lambda_{k-1}}^{\lambda_{k}}\left(\lambda-a_{k}\right)^{2} d n(\lambda) \leq\left(a_{k+1}-a_{k}\right)^{2}
$$

we get that for $|k-m|>1$.

$$
\begin{aligned}
\int_{\lambda_{k-1}}^{\lambda_{k}}\left(\ln \left|\frac{\lambda-x}{a_{k}-x}\right|-\ln \left|\frac{\lambda+x}{a_{k}+x}\right|\right) d n(\lambda) \mid \\
\leq \frac{2 a_{k} x\left(a_{k+1}-a_{k}\right)^{2}}{\left(a_{k}-x\right)^{2}\left(a_{k}+x\right)^{2}}+2 C_{1} \frac{\left|a_{k+1}-a_{k}\right|^{3}}{\left(a_{k}-x\right)^{3}} .
\end{aligned}
$$

Invoking (A1), (A2) and (4.6), we get that for $|k-m|>1$

$$
\begin{aligned}
& \left|\int_{\lambda_{k-1}}^{\lambda_{k}}\left(\ln \left|\frac{\lambda-x}{a_{k}-x}\right|-\ln \left|\frac{\lambda+x}{a_{k}+x}\right|\right) d n(\lambda)\right| \\
& \leq C_{2}\left(\frac{1}{k} \cdot \frac{\lambda_{k}^{3} x}{\left(a_{k}-x\right)^{2}\left(\lambda_{k}+x\right)^{2}}+\frac{1}{k^{3 / 2}} \cdot \frac{\lambda_{k}^{3}}{\left|a_{k}-x\right|^{3}}\right)
\end{aligned}
$$

where $C_{2}<\infty$ is a constant not depending on $k$ and $m$. For $x \in\left[\lambda_{m-1}, \lambda_{m}\right]$ and $|k-m|>1$ the numbers $\left|a_{k}-x\right|$ and $\left|\lambda_{k}-\lambda_{m}\right|$ are of the same order of magnitude. In particular,

$$
\frac{1}{\left|a_{k}-x\right|} \leq C_{3} \frac{1}{\left|\lambda_{k}-\lambda_{m}\right|}
$$

where $C_{3}$ is independent of $k$ and $m$. From this and (5.6) (see also (5.3)) we derive the inequality

$$
\begin{aligned}
& \left|\sum_{\substack{k \in \mathbb{N} \\
|k-m|>1}}\left[\ln \left|b_{k}(x)\right|-V_{k}(x)\right]\right| \\
& \quad \leq C_{4} \sum_{\substack{k \in \mathbb{N} \\
|k-m|>1}}\left(\frac{1}{k} \cdot \frac{\lambda_{k}^{3} \lambda_{m}}{\left|\lambda_{k}-\lambda_{m}\right|^{2}\left(\lambda_{k}+\lambda_{m}\right)^{2}}+\frac{1}{k^{3 / 2}} \cdot \frac{\lambda_{k}^{3}}{\left|\lambda_{k}-\lambda_{m}\right|^{3}}\right)
\end{aligned}
$$

where $C_{4}$ is independent of $x$ and $m$.

Let us show that the sum on the right-hand side of (5.7) is bounded above, with a bound which is independent of $m$ (and hence, independent of $x$ ). Using (4.6), it is not difficult to check that

$$
\frac{1}{\left|\lambda_{k}-\lambda_{m}\right|} \leq \begin{cases}C_{5} \frac{1}{\lambda_{m}} & \text { if } k \leq m-\sqrt{m} \\ C_{5} \frac{1}{\lambda_{k}} & \text { if } k \geq m+\sqrt{m} \\ C_{5} \frac{1}{\lambda_{m}} \sqrt{m-k} & \text { if } m-\sqrt{m}<k<m+\sqrt{m}\end{cases}
$$


where $C_{5}<\infty$ is independent of $k$ and $m$. From these estimates we derive that for every $m \in \mathbb{N}$

$$
\begin{aligned}
& \sum_{\substack{k \in N \\
|k-m|>1}} \frac{1}{k} \cdot \frac{\lambda_{k}^{3} \lambda_{m}}{\left|\lambda_{k}-\lambda_{m}\right|^{2}\left(\lambda_{k}+\lambda_{m}\right)^{2}} \\
& \quad \leq C_{5}^{2}\left(\sum_{k \leq m-\sqrt{m}} \frac{1}{k}\left(\frac{\lambda_{k}}{\lambda_{m}}\right)^{3}+\sum_{k \geq m+\sqrt{m}} \frac{1}{k} \frac{\lambda_{m}}{\lambda_{k}}+\sum_{\substack{m \\
m-\sqrt{m}|k<m<m+\sqrt{m}\\
| k-m \mid>1}} \frac{1}{k} \frac{\lambda_{k}^{2}}{\lambda_{m}^{2}} \frac{m}{(m-k)^{2}}\right) .
\end{aligned}
$$

Analogously,

$$
\begin{aligned}
& \sum_{\substack{k \in \boldsymbol{N} \\
|k-m|>1}} \frac{1}{k^{3 / 2}} \cdot \frac{\lambda_{k}^{3}}{\left|\lambda_{k}-\lambda_{m}\right|^{3}} \\
& \leq C_{5}^{3}\left(\sum_{k \leq m-\sqrt{m}} \frac{1}{k^{3 / 2}} \frac{\lambda_{k}^{3}}{\lambda_{m}^{3}}+\sum_{k \geq m+\sqrt{m}} \frac{1}{k^{3 / 2}}+\sum_{\substack{m-\sqrt{m<k<m+\sqrt{m}}|k-m|>1}} \frac{\lambda_{k}^{3}}{\lambda_{m}^{3}} \frac{m^{3 / 2}}{k^{3 / 2}} \frac{1}{|m-k|^{3}}\right) .
\end{aligned}
$$

Using the expression (4.6) for $\lambda_{k}$, it is not difficult to see that the sums on the righthand sides of the last two big inequalities are bounded by a constant not depending on $m$. From here and from (5.7) it follows that (still assuming $x \in\left[\lambda_{m-1}, \lambda_{m}\right]$ )

$$
\left|\sum_{\substack{|k \in N\\| k-m \mid>1}}\left[\ln \left|b_{k}(x)\right|-V_{k}(x)\right]\right| \leq C_{6}
$$

with $C_{6}$ independent of $x$ and $m$.

Now by (5.4) and (4.10) we may write

$$
|\ln | B_{p}(x)\left|-V(x)-\sum_{k=m-1}^{m+1}\left[\ln \left|b_{k}(x)\right|-V_{k}(x)\right]\right| \leq C_{7}
$$

where $C_{7}<\infty$ is independent of $x$ and $m$. Thus, estimating $\ln \left|B_{p}(x)\right|-V(x)$ has been reduced to estimating the sum of three terms. Since (see (5.5) and (A2)) for $|k-m| \leq 1$

$$
\left|\frac{\lambda+x}{a_{k}+x}-1\right| \leq \frac{a_{k}-a_{k-1}}{a_{k}+x}=\frac{1}{4 \omega \sqrt{m}}(1+o(1))
$$

as $\dot{m} \rightarrow \infty$, we have

$$
\lim _{x \rightarrow \infty} \sum_{|k-m| \leq 1} \int_{\lambda_{k-1}}^{\lambda_{k}} \ln \left|\frac{\lambda+x}{a_{k}+x}\right| \ln (\lambda)=0
$$


From here, taking into account (5.3), we derive that

$$
|\ln | B_{p}(x)\left|-V(x)-\left(\sum_{k=m-1}^{m+1} \ln \left|x-a_{k}\right|-\int_{\lambda_{m-2}}^{\lambda_{m+1}} \ln |\lambda-x| d n(\lambda)\right)\right| \leq C_{8}
$$

where $C_{8}<\infty$ does not depend on $x$ and $m$. We estimate the difference

$$
\sum_{m-1 \leq k \leq m+1} \ln \left|x-a_{k}\right|-\int_{\lambda_{m-2}}^{\lambda_{m+1}} \ln |\lambda-x| d n(\lambda)
$$

by looking at the sum and at the integral separately. Although the integral here cannot be calculated explicitly, it can be estimated easily. We use the expression (4.3) for $n(\lambda)$ :

$$
\begin{aligned}
& \int_{\lambda_{m-2}}^{\lambda_{m+1}} \ln |x-\lambda| d n(\lambda)=2 \omega^{2} \int_{\lambda_{m-2}}^{\lambda_{m+1}} \ln |x-\lambda| \frac{\ln \lambda}{\lambda} d \lambda \\
& =2 \omega^{2} \int_{\substack{\lambda:|\lambda-x| \geq 1 \\
\lambda \in\left[\lambda_{m}-2, \lambda_{m+1}\right]}} \ln |x-\lambda| \frac{\ln \lambda}{\lambda} d \lambda+o(1) .
\end{aligned}
$$

Because

$$
\frac{\ln \lambda_{m+1}}{\lambda_{m+1}} \leq \frac{\ln \lambda}{\lambda} \leq \frac{\ln \lambda_{m-2}}{\lambda_{m-2}} \quad\left(x \in\left[\lambda_{m-2}, \lambda_{m+1}\right]\right)
$$

and $\ln |x-\lambda| \geq 0$

$$
\begin{aligned}
2 \omega^{2} \frac{\ln \lambda_{m+1}}{\lambda_{m+1}} & \int_{\lambda_{m-2}}^{\lambda_{m+1}} \ln |x-\lambda| d \lambda+o(1) \\
& \leq \int_{\lambda_{m-2}}^{\lambda_{m+1}} \ln |x-\lambda| d n(\lambda) \\
& \leq 2 \omega^{2} \frac{\ln \lambda_{m-2}}{\lambda_{m-2}} \int_{\lambda_{m-2}}^{\lambda_{m+1}} \ln |x-\lambda| d \lambda+o(1)
\end{aligned}
$$

for $x \in\left[\lambda_{m-1}, \lambda_{m}\right]$. The last integral can be calculated:

$$
\int_{\lambda_{m-2}}^{\lambda_{m+1}} \ln |x-\lambda| d \lambda=\left(\lambda_{m+1}-x\right) \ln \left(\lambda_{m+1}-x\right)+\left(x-\lambda_{m-2}\right) \ln \left(x-\lambda_{m-2}\right) .
$$


Here $x \in\left[\lambda_{m-1}, \lambda_{m}\right]$, and hence

$$
\begin{gathered}
\frac{1}{3}\left(\lambda_{m}-\lambda_{m-1}\right) \leq \lambda_{m+1}-x \leq 3\left(\lambda_{m}-\lambda_{m-1}\right) \\
\frac{1}{3}\left(\lambda_{m}-\lambda_{m-1}\right) \leq\left(x-\lambda_{m-2}\right) \leq 3\left(\lambda_{m}-\lambda_{m-1}\right) .
\end{gathered}
$$

Thus,

$$
\begin{aligned}
& \left|\left(\lambda_{m+1}-x\right) \ln \left(\lambda_{m+1}-x\right)-\left(\lambda_{m+1}-x\right) \ln \left(\lambda_{m}-\lambda_{m-1}\right)\right| \leq 3 \ln 3\left|\lambda_{m}-\lambda_{m-1}\right| \\
& \left|\left(x-\lambda_{m-2}\right) \ln \left(x-\lambda_{m-2}\right)-\left(x-\lambda_{m-2}\right) \ln \left(\lambda_{m}-\lambda_{m-1}\right)\right| \leq 3 \ln 3\left|\lambda_{m}-\lambda_{m-1}\right| .
\end{aligned}
$$

From here and from (5.9) it follows that for $x \in\left[\lambda_{m-1}, \lambda_{m}\right]$,

$$
\int_{\lambda_{m-2}}^{\lambda_{m+1}} \ln |x-\lambda| d \lambda-\delta_{m}|\leq 6 \ln 3| \lambda_{m}-\lambda_{m-1} \mid
$$

where $\delta_{m}=\left(\lambda_{m+1}-\lambda_{m-2}\right) \ln \left(\lambda_{m}-\lambda_{m-1}\right)$. From this and (5.8) we derive the inequalities

$$
\begin{aligned}
2 \omega^{2} \frac{\ln \lambda_{m+1}}{\lambda_{m+1}} \delta_{m} & -12 \omega^{2} \ln 3 \frac{\ln \lambda_{m+1}}{\lambda_{m+1}}\left(\lambda_{m}-\lambda_{m-1}\right) \\
& \leq \int_{\lambda_{m-2}}^{\lambda_{m+1}} \ln |x-\lambda| \operatorname{dn}(\lambda) \\
& \leq 2 \omega^{2} \frac{\ln \lambda_{m-2}}{\lambda_{m-2}} \delta_{m}+12 \omega^{2} \ln 3 \frac{\ln \lambda_{m-2}}{\lambda_{m-2}}\left(\lambda_{m}-\lambda_{m-1}\right) .
\end{aligned}
$$

Since the values

$$
\frac{\ln \lambda_{m-2}}{\lambda_{m-2}}\left(\lambda_{m}-\lambda_{m-1}\right) \quad \text { and } \quad \frac{\ln \lambda_{m+1}}{\lambda_{m+1}}\left(\lambda_{m}-\lambda_{m-1}\right)
$$

are bounded as $m \rightarrow \infty$, we get

$$
2 \omega^{2} \frac{\ln \lambda_{m+1}}{\lambda_{m+1}} \delta_{m}+O(1) \leq \int_{\lambda_{m-2}}^{\lambda_{m+1}} \ln |x-\lambda| d n(\lambda) \leq 2 \omega^{2} \frac{\ln \lambda_{m-2}}{\lambda_{m-2}} \delta_{m}+O(1) .
$$

Using the expression (4.6) for $\lambda_{k}$, we obtain

$$
2 \omega^{2} \frac{\ln \lambda_{m-2}}{\lambda_{m-2}}\left(\lambda_{m+1}-\lambda_{m-2}\right)=3+O\left(\frac{1}{\ln \lambda_{m}}\right) \quad(m \rightarrow \infty) .
$$


Thus, for $m \rightarrow \infty$ we have the asymptotic estimates

$$
\begin{aligned}
& 2 \omega^{2} \frac{\ln \lambda_{m-2}}{\lambda_{m-2}} \delta_{m}=3 \ln \left(\lambda_{m}-\lambda_{m-1}\right)+O(1) \\
& 2 \omega^{2} \frac{\ln \lambda_{m+1}}{\lambda_{m+1}} \delta_{m}=3 \ln \left(\lambda_{m}-\lambda_{m-1}\right)+O(1) .
\end{aligned}
$$

From these and (5.11) it follows that

$$
\left|\int_{\lambda_{m-2}}^{\lambda_{m+1}} \ln \right| x-\lambda|\operatorname{dn}(\lambda)-3 \ln | \lambda_{m}-\lambda_{m-1}|| \leq C_{9}
$$

where $C_{9}$ is a constant not depending on $x$ and $m$. Further, since

$$
\left|x-a_{k}\right| \leq 4\left(\lambda_{m}-\lambda_{m-1}\right) \quad(k=m-1, m, m+1)
$$

we have

$$
\sum_{k=m-1}^{m+1} \ln \left|x-a_{k}\right| \leq 3 \ln \left(\lambda_{m}-\lambda_{m-1}\right)+3 \ln 4 .
$$

On the other hand, if $x \in\left[\lambda_{m-1}, \lambda_{m}\right]$ and $\left|x-a_{m}\right| \geq \varepsilon\left|a_{m}-a_{m-1}\right|$, then

$$
\left|x-a_{m+1}\right| \geq \frac{1}{4}\left|\lambda_{m}-\lambda_{m-1}\right| \quad \text { and } \quad\left|x-a_{m-1}\right| \geq \frac{1}{4}\left|\lambda_{m}-\lambda_{m-1}\right|
$$

and hence

$$
\sum_{k=m-1}^{m+1} \ln \left|x-a_{k}\right| \geq 3 \ln \left(\lambda_{m}-\lambda_{m-1}\right)-3 \ln 4-\ln \varepsilon .
$$

From (5.12), (5.13) and (5.10) we derive the estimates

$$
\sum_{k=m-1}^{m+1} \ln \left|x-a_{k}\right|-\int_{\lambda_{m-2}}^{\lambda_{m+1}} \ln |x-\lambda| \operatorname{dn}(\lambda)\left\{\begin{array}{l}
\leq C_{10} \\
\geq-C_{10}-\ln \varepsilon
\end{array}\right.
$$

valid for $|x-a| \geq \varepsilon\left(a_{m}-a_{m-1}\right)$, where $C_{10}<\infty$ is a constant not depending on $x, m$ and $\varepsilon$. Comparing the last estimate with (5.12), we get the inequalities

$$
\ln \left|B_{p}(x)\right| \begin{cases}\leq V(x)+C_{11} & \text { if } x \in(0, \infty) \\ \geq V(x)-C_{11}-\ln \varepsilon & \text { if } x \in(0, \infty) \backslash N(\varepsilon)) .\end{cases}
$$

These, together with (4.5) give the desired estimates (4.1) and (4.2). 


\section{References}

[1] Achieser, N. I. and I. M. Glasmann: Theorie der linearen Operatoren im Hilbert-Raum. Berlin: Akademie-Verlag 1981.

[2] Burckel, R. B.: An Introduction to Classical Complex Analysis. Volume I. New York: Academic Press 1979.

[3] Doetsch, G.: Handbuch der Laplace-Transformation. Volume I. Basel: Birkhäuser Verlag 1950.

[4] Duren, P. L.: Theory of $H^{p}$ Spaces. New York: Academic Press 1970.

[5] Rudin, W.: Real and Complex Analysis. New York: McGraw-Hill 1966.

[6] Salamon, D.: Realization theory in Hilbert space. Math. Systems Theory 21 (1989), 147 - 164.

[7] Salamon, D.: Infinite dimensional systems with unbounded control and observation: a functional analytic approach. Trans. Amer. Math. Soc. 300 (1987), 383 - 431.

[8] Weiss, G.: Admissible observation operators for linear semigroups. Israel J. Math. 65 (1989), $17-43$.

[9] Weiss, G.: Transfer functions of regular linear systems. Part I: Characterizations of regularity. Trans. Amer. Math. Soc. 342 (1994), 827 - 854.

[10] Weiss, G.: Regular linear systems with feedback. Math. Control, Signals and Systems 7 (1994), 23 - 57.

Received 20.03.1995 